\title{
Diskussion
}

\author{
Elsbeth Kneuper
}

\section{Manche Frauen haben keine Kinder. Vergleichende Bemerkungen aus der Medizinethnologie}

Westliche Versicherungssysteme ermöglichen es Frauen und Männern, zwischen einem Leben mit und ohne Kinder zu wählen, ohne um ihre Altersversorgung fürchten zu müssen. Als Gegenbild zu diesem Raum, den ich im Folgenden bei aller Vorsicht, die bei solchen Verallgemeinerungen geboten ist, mit Margaret Lock $(2000,234)$ »Euroamerika« nennen werde, erscheinen nach wie vor die so genannten Entwicklungsländer. Weil dort die Altersversorgung wesentlich von eigenen Kindern abhängt oder abzuhängen scheint, liegt für uns die Annahme nahe, dass Kinderlosigkeit dort keine Option für Frauen darstellt. Das führt zu der Vorstellung, dass nicht nur ein wesentlicher Teil der Lebensarbeit von Frauen außerhalb Euroamerikas in Gebären und Aufziehen von Kindern besteht, sondern dass das geradezu ihre natürliche Aufgabe ist.

Diese Vorstellung ist nicht einfach nur ein verbreitetes Vorurteil, sondern wirkt in die Entwicklungspolitik und hier insbesondere in die öffentliche Gesundheitsversorgung hinein. Die einseitige Konzeption dieser Gesellschaften als Empfänger von Entwicklungshilfe verbaut uns den Blick auf deren kreativen Umgang mit sozialen Bedingungen und macht sie zur Projektionsfläche eigener regressiver Geschlechterrollen. Für eine Relativierung nicht nur unserer eigenen Ideen von Geschlecht, sondern auch des latent evolutionistischen Bildes anderer Kulturen, denen diese Ideen unterlegt werden, müssen wir uns klar machen, wie entwick- lungsgesundheitspolitische Maßnahmen wirken und warum diese mit indigenen Vorstellungen aktuell oder latent im Konflikt stehen. Denn auch in den so genannten Entwicklungsländern sind Frauen weder durchweg auf das Gebären angewiesen, um eigene Nachfahren zu bekommen, noch sind eigene Kinder generell für ein gelungenes Leben von Frauen notwendig.

\section{I.}

Die Folgen unserer Sicht auf die "Entwicklungsländer" kann man am Beispiel Ägyptens illustrieren. "There are hopeful signs", bemerkt Carol MacCormack, "that women as women are becoming visible in health research. In the United States, Canada, and most of Europe, for example, women's health has become a political issue" $(1996,335)$. Im Kontrast dazu werden gesundheitliche Belange von Frauen in Ländern wie Ägypten immer noch im Wesentlichen unter reproduktiver $\mathrm{Ge}-$ sundheit rubriziert. Das hat allerdings nichts mit "traditionellen" ägyptischen Medizinen oder einem historisch gewachsenen Gesundheitssystem zu tun. Die renommierte ägyptische Medizinanthropologin Soheir Morsy macht darauf aufmerksam, dass es vor allem Programme der westlich bzw. US-amerikanisch dominierten internationalen Gesundheitspolitik sind, die Frauengesundheit auf reproduktive Gesundheit reduzieren und 
darüber Gesundheitsrisiken, die nicht unmittelbar im Zusammenhang mit Schwangerschaft und Geburt stehen, vernachlässigen. In Übereinstimmung mit den Vorgaben der internationalen Gesundheitspolitik und unter dem Einfluss von Programmen der WHO identifizieren ägyptische Ärzte solche Krankheiten als wichtigstes $R$ isiko der reproduktiven Gesundheit, die unmittelbar im Zusammenhang mit Schwangerschaft und Geburt stehen (Morsy 1995, 164). Hier kommt nicht einmal die etwas weiter gefasste maternal health, zu der etwa infektiöse oder parasitäre Erkrankungen sowie Kreislauferkrankungen gehören, in den Blick. Die Gesundheit von Frauen als Müttern wird nicht während ihrer gesamten reproduktiven Phase gesehen, sondern nur im direkten Umfeld der Schwangerschaft. Aber die Gesundheitsrisiken von Frauen gehen durchaus weiter: Die gleichen Krankheiten können Frauen auch dann befallen, wenn sie sich noch nicht oder nicht mehr im reproduktiven Alter befinden, ein Tatbestand, den die WHO ganz außen vor lässt. Wiederholt werden Erkrankungen des Kreislauf-, des Verdauungs-, des Atem- und des Urogenitalsystems als Ursachen von Müttersterblichkeit unberücksichtigt gelassen (El-Mouelhy 1987, Saleh, Gadalla und Fortney 1987 und Zurayk 1991).

Der hier diagnostizierte blinde Fleck der internationalen Gesundheitsvorsorge betrifft im Übrigen auch Teile der feministischen Kritik, die sich ebenfalls auf die reproduktive Gesundheit konzentrieren. ${ }^{1}$ Dabei wäre deren Aufgabe durchaus weiter zu fassen. "While reproductive health continues to be the primary focus of hegemonic international feminist discourse, state-sanctioned ,development programs which threaten public health, i.e., the health of women, children, and men, remain relatively neglected. These programs are rarely the objects of analytical scrutiny in the sociomedical literature on Egypt" (Morsy 1998, 80). Hierher gehören nicht nur die Anwendung von hochgiftigen Pestiziden oder der Import von radioaktiv verseuchten Nahrungsmitteln, sondern auch die Tatsache, dass es internationale Institutionen unter Einfluss des Westens waren, die die Privatisierung des ägyptischen Gesundheitssystems auf Kosten der Armen durchgesetzt haben (ebd. 81). Der Umstand, dass "preferential consideration is given to women's reproductive health, studies of health service utilization and individual therapeutic narratives, and occasional self-congratulatory accounts of expatriate do-gooders bearing the torch of enlightenment" (ebd. 80), muss daher auf die informierte Beobachterin zynisch wirken.

Die Konzentration auf die reproduktive Gesundheit bei der öffentlichen Gesundheitsversorgung von Frauen im Rahmen internationaler Programme ist also ein Import des Westens. Nicht die emische Sicht kommt hier zum Tragen, sondern das Frauenbild der internationalen Institutionen, die hier wirken. Es sind also westliche Ansichten über Länder wie Ägypten, deren Folgen wir hier beobachten können.

Damit ist selbstverständlich noch nicht gezeigt, dass Frauen in nicht westlichen Gesellschaften nicht dennoch im Wesentlichen darauf festgelegt sind, Kinder $z u$ gebären und großzuziehen. Aber es gibt auch in nicht westlichen Gesellschaften Entwürfe für ein gelungenes Leben von Frauen, die keine Kinder gebären. Das lässt sich mit Hilfe von ethnologischem Material etwa aus Forschungen bei den Gikuyu oder den Plains-Indianern bele-

Eine angemessen umfangreiche Diskussion des Sachverhalts in der Sozialanthropologie findet sich etwa bei Page, Helan E. (1994): Breaking the silence on violence in academia. 
gen. Beim Thema Kinderlosigkeit bieten diese beiden Fälle zwei besonders interessante komplementäre Modelle, bei denen das eine kompensatorisch mit Kinderlosigkeit umgeht, während im anderen Kinderlosigkeit als implizite Folge von elastischen Rollenskripts gewollt oder zumindest akzeptiert wird.

\section{II.}

Die Gikuyu leben zum überwiegenden Teil in Kenia. Elisabeth Tietmeyer hat die Lebenswelt der Ethnie in mehreren Feldforschungen untersucht und detailliert beschrieben. Sie sind virilokal, patrilinear und kennen Polygamie. Zentrale Bedeutung für ihr Verwandtschaftssystem der vorkolonialen Zeit, die "Organisation" ihres Alltags und ihre Kosmologie hat der Umstand, dass Menschen in ihren jeweiligen Enkeln reinkarniert werden. In ihren Enkeln erkennen sich die Gikuyu jeweils selbst. In präkolonialer Zeit "erhielt der erste Sohn den Namen seines Großvaters väterlicherseits, der zweite Sohn den $\mathrm{Na}$ men seines Großvaters mütterlicherseits; die erste Totcher wurde nach der Mutter ihres Vaters benannt, die zweite Tochter nach der Mutter ihrer Mutter. Alle weiteren Kinder bekamen ebenfalls alternierend den Namen des seinem biologischen Geschlecht entsprechenden elterlichen Geschwisters" (Tietmeyer 1998, 165). Die sich hierin ausdrückende kosmologisch begründete Beziehung ist nach Tietmeyer der Grund, "warum die Gikuyu alles erdenklich Mögliche unternahmen, um Kinderlosigkeit zu vermeiden" (ebd.).

Allerdings ist für die Gikuyu der biologische Aspekt der Deszendenz nicht entscheidend. Traditionell kann für einen Mann, der zeugungsunfähig ist, ein anderer Mann mit dessen Frauen Kinder zeugen, deren sozialer Vater der erste ist. Auch für Frauen gibt es im Falle von
Kinderlosigkeit einen Ausweg - die Gynägamie.

Die Gynägamie war eine soziale Institution, die sich bei mehreren afrikanischen Ethnien findet (Smith Oboler 1980, 69-88) und hier anhand des Beispiels der Gikuyu illustriert werden soll. Kinderlose Frauen hatten bei den präkolonialen Gikuyu die Möglichkeit, im Namen eines fiktiven Sohnes eine jüngere Frau zu heiraten, die der älteren Frau zu eigenen Nachkommen verhelfen sollte. Diese älteren Frauen, zumeist Witwen, nahmen den jüngeren gegenüber die Position der Schwiegermutter, aber zugleich zusammen mit dem Genitor ihres zukünftigen Nachwuchses die eines Ehemannes ein. Die Ältere wählte den Genitor aus, wie ein Ehemann zahlte sie den Brautpreis und baute der jüngeren Frau eine Hütte in ihrem Gehöft. Beide Frauen hatten gegeneinander eheliche Pflichten: die Ältere hatte alle Zeremonien und Riten zu organisieren, die im Zusammenhang mit der Jüngeren und den gemeinsamen Kindern entstanden, die Jüngere musste den Haushalt versorgen, Kinder gebären und großziehen.

Die Kinder nannten die ältere "Großmutter", und ihr erster Name wies sie als deren Enkel aus, d.h. die älteste Tochter trug ihren Namen, der älteste Sohn den Namen ihres - eventuell verstorbenen - Mannes. Die Position der Älteren als Ehemann der Jüngeren drückte sich im zweiten Namen der Frau und der Kinder aus. Tietmeyer nennt ein Beispiel: Die Kinder aus einer gynägamen Beziehung, in der die Ältere Wanjiru hieß, "trugen nicht den Namen des fiktiven Mannes, sondern den der älteren Frau (z. B. )Ngugi wa Wanjiru، = ,Ngugi, Sohn von Wanjirus). Dies zeigte an, dass die jüngere Frau und die Kinder zu einer gynägamen Familie gehörten" (Tietmeyer 1998, 172). Das Verwachsen mit der männlichen Rolle gestattet in einer patri- 
linearen Gesellschaft die Namensweitergabe und damit eigene Nachkommenschaft für die Fortexistenz in eigenen Enkeln.

Die Kolonialisierung und der Einfluss des Westens haben die Gikuyu-Kultur verändert. Die Gynägamie hat sich erhalten, aber ihre Funktion gewechselt. Während der Glaube an die Reinkarnation an Bedeutung verloren hat, spielen wirtschaftliche Beweggründe eine größere Rolle. Weil Frauen heute anders als in der präkolonialen Zeit eigenes Land besitzen und vererben können, wird die Gynägamie für sie zu einem Mittel, zu verhindern, dass ihr Besitz etwa an die Familie ihres verstorbenen Mannes fallt. Vor allem wohlhabende Frauen sichern sich auf diesem Wege Hilfe für den Haushalt und die Feldarbeit und eine Versorgung furr das Alter. Die jungen Frauen, die eine solche Beziehung eingehen, haben meist uneheliche Kinder und können sich durch die Beziehung zu der Älteren aus der daraus resultierenden präkeren ökonomischen Situation befreien. Die Beziehung ist auch heute noch rechtlich verbindlich und kann, wenn eine der Parteien ihren Pflichten nicht nachkommt, von einem staatlichen Gericht geschieden werden (Tietmeyer 1998, 174).

Diese Skizze der Gynägamie bei den Gikuyu zeigt, dass eine Frau Nachkommen haben kann, ohne Kinder zu gebären. Während ihre klassifikatorischen Enkel aus einer gynägamen Beziehung in vorkolonialer Zeit immer zugleich Enkel ihres Mannes waren, kann eine GikuyuFrau heute eine eigene Familie ohne Mann gründen. Reproduktive Gesundheit im Sinne der öffentlichen Gesundheitsversorgung spielt für sie, obwohl sie für ihre Altersversorgung auf Nachkommenschaft angewiesen ist, keine Rolle schon deshalb, weil der Umstand, dass sie keine Kinder geboren hat, nicht gesundheitliche Gründe haben muss. Insbeson- dere gilt für sie nicht, dass die Arbeit im Zusammenhang mit Reproduktion ihr überlassen ist. Zudem erweist sich angesichts dieses Beispiels die Erwartung, dass das Gebären von Kindern die je eigene Altersversorgung sichert, als grobe Vereinfachung. Gerade die Kinderlosigkeit der Älteren und die dadurch mögliche ökonomische Unabhängigkeit stellt in einer gynägamen Beziehung das Kapital dar, durch das diese fuir ihre Partnerin interessant ist. Damit wird eine grundlegende Annahme über die Bedeutung von Kindern entschieden relativiert. Allerdings ist eine Gikuyu-Frau, die in einer gynägamen Beziehung die Ältere ist, nicht "kinderlos«. Die Gynägamie ist ja vielmehr gerade ein Mittel, ihr Kinder bzw. Enkel zu verschaffen.

Indessen gibt es auch Beispiele von Ethnien, in denen soziale Positionen existieren, für die Kinderlosigkeit erwünscht ist oder zumindest als notwendige Folge angesehen wird. Ein Fall ist die von der westlichen Ethnologie als "Berdache« bezeichnete Institution bei den nordamerikanischen Indianern. Die US-amerikanische Ethnologin Harriet Whitehead hat anhand historischen Materials den entscheidenden Schritt zu einer umfassenden Deutung des Phänomens getan (1981). Für die Ethnien, die vor der Ankunft der Europäer Nordamerika besiedelten, hatte geschlechtliche Identität zwei Aspekte, einen anatomischen und einen habituellen. Whitehead spricht statt vom habituellen Aspekt der Geschlechtsidentität gelegentlich vom sozialen Geschlecht. Das scheint mir zwar nicht falsch, aber doch irreführend, insofern die Betonung der Anatomie und - dazu später Näheres - der Menstruation selbstverständlich auch sozialen Konventionen und Wissensproduktionen entspringt (Buckley/Gottlieb 1988, 40-50).

Bei der Geburt entscheidet zunächst die Anatomie darüber, welchem Ge- 
schlecht ein Kind zugeordnet wird. Damit ist die geschlechtliche Identität des Kindes indessen noch nicht endgültig festgelegt. Die zweite Determinante des Geschlechts äußert sich im Verhalten, in Vorlieben für Spielzeug oder geschlechtsspezifische Beschäftigungen und Kleidung. Während anatomisches und habituelles Geschlecht in den meisten Fällen kongruieren, kann es vorkommen, dass Kinder Verhalten und Vorlieben an den Tag legen, die ihrem anatomischen Geschlecht nicht entsprechen. "The wergen", schreibt Kroeber über die Berdache der Yurok, "usually manifested the first symptoms of his proclivities by beginning to weave baskets. Soon he donned women's clothing and pounded acorns" (Kroeber 1925, 46). Auch Träume scheinen eine Rolle zu spielen: "Supplementing and sometimes substituting for youthful behavioral cues were messages that come to future gender-crosser in dreams and visions" (Whitehead 1981, 86). Erweist sich diese Disposition als permanent, kann das Kind den Status eines Berdache erlangen und Aspekte zweier Geschlechter in sich vereinen. Es wird zu etwas Intermediären, das als "Frau-Mann", "halb Frau, halb Mann" oder "nicht Mann, nicht Frau " umschrieben wird (ebd. 88). Damit lassen sich vier verschiedene geschlechtliche Identitäten ausmachen: Es gibt die zwei Fälle, bei denen Verhalten und Anatomie kongruieren, die ich im Folgenden der Einfachheit halber und soweit es nicht zu Missverständnissen kommen kann "Frauen" bzw. "Männer" nennen werde. Und es gibt anatomische Männer, die habituell Frauen sind und anatomische Frauen, die habituell Männer sind, beide werden im Weiteren "Berdache" genannt.

Vor allem für anatomische Männer ist dieser Status als eigenständige geschlechtliche Identität gut dokumentiert. Seine Betätigungsfelder als Berdache deckten sich im Großen und Ganzen mit denen von Frauen. Dennoch konnten sie oft auch bestimmte männliche Tätigkeiten ausüben. Die Navajo schätzten die "gender-crosser" (Whitehead 1981, 89), weil sie die Aufgaben beider Geschlechter übernehmen konnten, und erhoben sie nicht selten zu Haushaltsvorständen, wovon sie sich eine Garantie zukünftigen Wohlstands versprachen. Bei den Crow, den Papogo oder den Cheyenne gab es zudem rituelle Funktionen, die nur von Berdache ausgefuillt werden konnten.

Diese Sonderstellung belegt, dass es bei der Konzeption des Geschlechterunterschiedes neben einem hierarchischen - Frauen waren Männern untergeordnet auch ein komplementäres Moment gab: diejenigen, die eine mittlere Position einnahmen, verfügten gegenüber den Frauen und Männern über besondere Fähigkeiten.

Daraus lässt sich indessen nicht auf eine Symmetrie der Geschlechter schlieBen. So gab es sehr viel weniger anatomisch weibliche Berdache. Der Grund dafür, argumentiert Whitehead, liegt im anatomischen Aspekt der Geschlechtsidentität: Noch vor den Genitalien scheint die Regelblutung definierenden Charakter für die geschlechtliche Identität gehabt zu haben. Whitehead stellt fest: "Female blood and its attendant associations seems to have anchored women more firmly in their womanly identity than male anatomy anchored the man in his masculinity" (Whitehead 1981, 92). Obwohl der Wunsch von Mädchen, die männliche Rolle einzunehmen, bei den nordamerikanischen Indianern weit verbreitet war (ebd. 90) und Frauen z.B. gelegentlich in den Krieg zogen, war gender-crossing für Frauen nicht bei allen Ethnien möglich und kam generell seltener vor als bei Männern.

Es hat aber weibliche Berdache gegeben. Diese traten bei den südwestlichen 
Ethnien auf, bei den Mohave, den Cocopa, den Apachen und den Navajo. Was diese Ethnien von anderen unterschied, war die Vorstellung, dass mit dem Geschlechtswechsel zugleich eine Veränderung der Anatomie vor sich ging. Ebenso wie anatomisch männliche Berdache von ihrer Menstruation berichteten, wurde von anatomisch weiblichen Berdache angenommen, dass sie nicht menstruieren. Wie ihre anatomisch männlichen Pendants arbeiteten sie und kleideten sie sich entsprechend den Gewohnheiten des ihrer Anatomie komplementären Geschlechts. Insbesondere heirateten sie Frauen. Und das geht selbstverständlich mit eigener Kinderlosigkeit einher.

Das Beispiel der anatomisch weiblichen Berdache belegt, dass die Möglichkeit der Wahl, ein Leben zu führen, in dem Kinder nicht vorgesehen sind, nicht erst ein Verdienst christlicher Klöster oder der Aufklärung ist. Auch außerhalb des "Westens" gibt bzw. gab es institutionalisierte geschlechtliche Identitäten, die anatomischen Frauen Lebensentwürfe als Kinderlose ermöglichen. Dabei ist klarer Weise zuzugestehen, dass für die Berdache Kinderlosigkeit sekundär ist. Zudem ist die Wahl eines Lebensentwurfs hier nicht als Wahl einer autonomen Person im Sinne der Aufklärung zu verstehen.

Allerdings kommt sie dem überraschend nahe. So erklärt ein Informant der Mohave gegenüber George Devereux: "When there is a desire in a child's heart to become a transvestite, that child will act different. It will let people become aware of that desire. They may insist on giving the child the toys and garments of its true sex, but the child will throw them away" (Devereux 1937, 503). Es ist auch bei anderen Ethnien in der Regel das Kind, von dem der Wechsel der ge- schlechtlichen Identität ausgeht. ${ }^{2}$ Der Unterschied zu einer autonomen Entscheidung besteht darin, dass nordamerikanische Indianer Identität und damit handlungsleitende Dispositionen so konzipieren, dass sie von Geistern etwa in Träumen oder Visionen den Menschen überantwortet werden. Was Ruth Landes über die Anthropologie der Ojibwa schreibt, gilt mutatis mutandis für die nordamerikanischen Indianer insgesamt: "All those talents and traits of character which we think of as a function of a total personality are regarded by the Ojibwa as isolated, objective items which may be acquired in the course of life by individuals who are fortunate enough to coerce them from the supernatural. In Ojibwa thought, there is no original and absolute sself " (Landes 1938, 124). Dispositionen, Fähigkeiten und Charakterzüge werden zu Wirkungen von Instanzen, die außerhalb des Ich ihren Ursprung haben.

Mit dem Berdache-Status hielt die Gesellschaft der nordamerikanischen Indianer jedenfalls einen Lebensentwurf für Frauen bereit, in dem nicht vorgesehen war, dass sie Kinder gebären und großziehen, und zu dem ein Pendant in den euroamerikanischen Gesellschaften fehlt. Es ist in diesem Zusammenhang meines Erachtens im Übrigen keine bloß akademische Frage, ob wir die anatomisch männlichen Berdache nicht auch als kinderlose Frauen anzusehen hätten. Erworbene Aspekte der Identität haben bei den autochthonen Bewohnerinnen und Bewohnern Nordamerikas eine hohe Wertschätzung genossen. Insofern lässt es sich zumindest mit Blick auf die emische Sicht kaum rechtfertigen, den anatomischen Aspekt der geschlechtlichen Identität vor dem habituellen auszuzeichnen und anatomisch männliche Berdache

2 Das hat den Ethnopsychoanalytiker Devereux offenbar auch dazu verleitet, den Berdache-Status mit dem westlichen Konzept der Homosexualität zu identifizieren. 
umstandslos eine geschlechtliche Identität zuzuschreiben, die "eigentlich" männlich wäre. Die Wertschätzung anatomisch männlicher und habituell weiblicher Berdache hat dabei ganz handfeste Gründe. Von den Aufgaben der Kinderpflege entbunden, konnten sie in den weiblichen Tätigkeitsfeldern beträchtliches Geschick entwickeln. Insofern es die Frauen waren, die langlebige und nicht für den unmittelbaren Verbrauch bestimmte Güter wie Zelte, Decken etc. herstellten, konnten habituell weibliche Berdache zu einer Quelle von Wohlstands und Ansehen werden. Insofern sie oft, wenn auch nicht immer, kinderlos waren, sind sie daher meines Erachtens wenigstens »zur Hälfte" als kinderlose Frauen anzusehen.

Insbesondere für diese Berdache war ähnlich wie heute für die älteren Partnerinnen in gynägamen Beziehungen Kinderlosigkeit nicht ein Problem für die Altersversorgung, sondern ein Grund für materiellen Reichtum. Beide Beispiele belegen, dass der Universalismus der Biomedizin dazu tendiert, wichtige Unterschiede von Kulturen zu unterschlagen.

\section{III.}

Wenn Frauen Kinder haben, muss das, wie die Gynägamie bei den Gikuyu belegt, nicht unbedingt heißen, dass ihr Leben davon bestimmt ist, Kinder zu gebären und großzuziehen. Und wenn Frauen kinderlos sind, kann das sehr Unterschiedliches bedeuten, wie ein Vergleich des Westens mit den nordamerikanischen Ethnien zeigt. Frauen, die aus ökonomischen oder kosmologischen Gründen Nachkommen brauchen, müssen diese nicht notwendig selbst gebären. Und es kann erstrebenswerte gesellschaftliche Positionen geben, für die die Möglichkeit, eigene Kinder zu haben, nicht vorgesehen oder nebensächlich ist.
Die Möglichkeit, ohne Kinder ein erfülltes Leben $\mathrm{zu}$ führen, ist also auch außerhalb Euroamerikas gegeben. Zudem zeigt das Beispiel der Gikuyu, dass es durchaus nichtmedizinische Wege gibt, mit unerwünschter Kinderlosigkeit umzugehen. Vor diesem Hintergrund muss es erstaunen, dass Frauen in internationalen Programmen der öffentlichen Gesundheitsversorgung fast ausschließlich als potenzielle oder aktuelle Gebärende auftreten.

Ein Grund für die Reduktion von Frauen auf ihre reproduktive Funktion liegt in der Politik internationaler Institutionen, die Frauengesundheit allzu oft und vordringlich im Zusammenhang mit Geburtenkontrolle sehen. Das politische Anliegen - die Weltbank etwa hat Geburtenkontrolle immer wieder zu einer Bedingung für ökonomische Hilfe gemacht - wird zu einem gesundheitlichen: Eine Studie der Weltbank bspw. hat in diesem Sinne "excess fertility" als "disease in developing countries alongside malnutrition, anaemia, tetanus, AIDS" etc. identifiziert (Zurayk et al. 1992, 36).

Daneben spielt auch die Konstruktion des westlichen Selbstbildes als entwickelterer und fortschrittlicher Kultur eine Rolle. Indem wir die Reduktion von Frauen auf ihre Funktion als Gebärende und Erzieherin der Kinder nicht nur in unserer Vergangenheit finden oder zu finden meinen, sondern in die Gegenwart "unterentwickelter" Gesellschaften projizieren, bestätigen wir uns unsere eigene Position als besonders emanzipierter und entwickelter Gesellschaft. Dabei haften dem impliziten Universalismus der Biomedizin latente Residuen der Universalgeschichte an, die in dem, was für sie unterentwickelte Gesellschaften sind, eine "natürliche" Ordnung wirken sehen (Kneuper 2003).

Damit hat sich in der internationalen Gesundheitspolitik ein Frauenbild erhal- 
ten, das die westlichen Gesellschaften längst überwunden $\mathbf{z u}$ haben glaubten. Der Eindruck entsteht, als würden wir abgelegte Rollenbilder in die Gesellschaften der "Entwicklungsländer" exportieren, um uns selbst umso fortschrittlicher positionieren zu können. Das macht unser Engagement für die Frauen in diesen Ländern umso fragwürdiger. Und noch da, wo wir die genuinen Leistungen fremder Kulturen würdigen, geschieht das meist selektiv und ändert wenig an unseren vorgefertigten Vorstellungen (Kneuper 2003).

Entscheidend für einen Fortschritt nicht nur in der Gesundheitspolitik ist schließlich die Forderung, Frauen immer auch als potenziell kinderlos zu denken, denn Entwicklungsgesundheitspolitik kann sich nicht damit begnügen, das Leben von Frauen als Gebärende oder Mütter zu verbessern.

\section{Literatur}

Buckley, T./Gottlieb, A. (1988): A critical appraisal of theories of menstrual symbolism. In: Dies. (Hg.) (1988): Blood Magic: The Anthropology of Menstruation. Berkeley, S. 3-40.

Crouse, J. R. (1989): Gender, Lipoproteins, Diet, and Cardiovascular Risk: Sauce of the Goose May Not Be Sauce of the Gander. In: Lancet, Februar 11, S. 318-320

Devereux, George (1937): Institutionalized homosexuality of the Mohave Indians. In: $\mathrm{Hu}$ man Biology. Jg. 9, S. 498-527

El-Moulhy, Mawaheb (1987): Maternal Mortality in Egypt. Cairo

Hamilton, Jean (1992): Biases in Women's Health Research. In: Women and Therapy. Jg. 12, S. 91-101

Hamilton, Jean (1996): Women and Health Policy: On the Inclusion of Females in Clinic Trials. In: Sargent, Carolyn/Brettell, Caroline (Hrsg.): Gender and Health. An International Perspective. New Jersey, S. 292-325

Kneuper, Elsbeth (2003): Die inatürlicher Geburt - eine globale Errungenschaft? In Wolf, Angelika/Hörbst Viola (Hrsg): Medi- zin und Globalisierung: Universelle Ansprïche - lokale Antworten. Hamburg, S. 107-128

Kroeber, Alfred (1925): Handbook of the Indians of California. In: U.S. BAE Bulletin Jg. 78, S. $42-68$

Landes, Ruth (1938): The Objiwa Woman. New York

Lock, Margaret (2000): On dying twice: culture, technology and the determination of death. In: Dies. et al. (Hrsg.): New Medical Technologies. Intersections of Inquiry. Cambridge, S. 233-262

MacCormack, Carol (1996): Risk, Prevention, and International Health Policy. In: Sargent, Carolyn/Brettell, Caroline (Hrsg.): Gender and Health. An International Perspective. New Jersey, S. 326-337

Morsy, Soheir (1995): Deadly Reproduction among Egyptian Women: Maternal Mortality and the Medicalisation of Population Control'. In: Faye D. Ginsburg, Faye D./ Rapp, Rayna (Hrsg.): Conceiving the new World Order. The Global Politics of Reproduction. Berkeley, S. 162-176

Morsy, Soheir (1998): Not only women: science as resistance in open door Egypt. In: Lock, Margaret/Kaufert, Patricia (Hrsg.): Pragmatic women and body politics. Cambridge, S. 77-97

Saleh, Saneya/Saad, Gadalla/Fortney, Judith (1987): Maternal Mortality in Manoufia: a Study of Reproductive Age Mortality. Cairo

Smith Oboler, Regina (1980): Is the Female Husband a Man? Woman/Woman Marriage Among the Nandy of Kenya. In: Ethnology .Jg. 19, S.69-88

Tietmeyer, Elisabeth (1998): Geschlecht, Differenz und Gynägamie. Zur Multiplikation von Geschlechterrollen in Afrika. In: Hauser-Schäublin, Brigitta/Röttger-Rössler, Birgitt (Hrsg.): Differenz und Geschlecht. Neue Ansätze in der ethnologischen Forschung. Berlin, S. 163-183

Whitehead, Harriet (1981): The bow and the burden strap: a new look at institutionalized homosexuality in native North America. In: Ortner, Sherry B./Whitehead, Harriet (Hrsg.): Sexual Meanings. The cultural construction of gender and sexuality. Cambridge, S. $80-115$ 
Zurayk, Huda (1991): Study on reproductive morbidity in Rural Giza. Cairo, New Delhi.

Zurayk, Huda et al.: Reproductive Health and Reproductive Morbidity Concepts and Measures with Relevance to Middle Eastern Society. Unveröffentlichtes Manuskript 1992, zit. nach Morsy 1995.

\section{Florence Vienne}

\section{Die Geschichte der männlichen Sterilität schreiben - Das Beispiel der NS-Zeit}

In Masculin/Féminin. La pensée de la différence widmet die Anthropologin Françoise Héritier (1996) einen Teil ihrer Ausführungen der Bedeutung von Fruchtbarkeit und Unfruchtbarkeit im Denken über die Geschlechterdifferenz. Ihre Untersuchung zeigt, dass es zu allen Zeiten und in allen Kulturen und bis vor kurzem auch in der westlichen Welt immer die Frauen waren, die für die Kinderlosigkeit verantwortlich gemacht werden. Männer können zwar impotent und "geistig unfruchtbar" sein, körperliche Sterilität hingegen scheint in allen menschlichen $\mathrm{Ge}$ sellschaften als weibliche Angelegenheit betrachtet zu werden. Aus einer anderen - wissenschaftshistorischen - Perspektive weist Nelly Oudshoorn $(1994 ; 2003)$ darauf hin, dass die Reproduktionsmedizin im 20. Jahrhundert allein auf den Frauenkörper zentriert war. Den Grund hierfür sieht sie in dem Fehlen institutioneller Strukturen und Netzwerke, die sich mit den männlichen Geschlechtsorganen befassten. Während Ärzte in gynäkologischen Kliniken Zugang zu weiblichen Patienten hatten, gab es bis in die 1960er Jahre für Männer keine entsprechende medizinische Disziplin. Folgt man diesen Studien, so beginnt die Geschichte des Mannes als geschlechtspezifisches, (un)fruchtbares Wesen und Gegenstand einer ,Wissenschaft vom Manne erst in der zweiten Hälfte des 20. Jahrhunderts.

Bis zu den 1970er Jahren gab es tatsächlich keine Andrologie als eine institutionalisierte medizinische Disziplin, die sich mit der Physiologie und Pathologie der männlichen Reproduktion befasst. Trotzdem entwickelte sich von der Jahrhundertwende bis in die NS-Zeit eine medizinische Literatur über männliche (Un)fruchtbarkeit: Artikel zu Erektionsund Ejakulationsstörungen sowie über die Qualität von Spermien erschienen in allgemeinmedizinischen, gynäkologischen, urologischen und endokrinologischen Zeitschriften; etliche Monographien widmeten sich speziell der Erforschung und Behandlung der Sterilitas virilis. In meinem Forschungsprojekt möchte ich gerade diesen Diskurs in den Blick nehmen. Dabei verfolge ich ein zweifaches Ziel: Zum einen geht es darum zu zeigen, wie der Mann in seiner körperlichen Spezifizität Gegenstand medizinischem Wissens wurde. Zum anderen möchte ich die Gründe dafür erkunden, warum die Medikalisierung des Mannes in der bisherigen Geschichtsschreibung und $\mathrm{Ge}$ schlechterforschung nicht wahrgenommen wurde. Während die Sexualisierung und Pathologisierung der Frau u.a. auch durch die Gynäkologie weitgehend erforscht ist (vgl. u.a. Laqueur 1990; Honegger 1991; Mosucci 1990), ist die Geschichte des Mannes als Objekt medizinischen Wissens bisher ungeschrieben geblieben. Im Rahmen dieses kurzen Beitrags kann die Medikalisierung des 